University of Wollongong

Research Online

Australian Institute for Innovative Materials -

Papers

Australian Institute for Innovative Materials

$1-1-2014$

A solvothermal strategy: one-step in situ synthesis of self-assembled 3D graphene-based composites with enhanced lithium storage capacity

Jingjing $\mathrm{Ma}$

Shanghai Jiao Tong University

Jiulin Wang

Shanghai Jiao Tong University

Yu-Shi He

Shanghai Jiao Tong University

Xiao Zhen Liao

Shanghai Jiao Tong University

Jun Chen

University of Wollongong, junc@uow.edu.au

See next page for additional authors

Follow this and additional works at: https://ro.uow.edu.au/aiimpapers

Part of the Engineering Commons, and the Physical Sciences and Mathematics Commons

Research Online is the open access institutional repository for the University of Wollongong. For further information contact the UOW Library: research-pubs@uow.edu.au 


\title{
A solvothermal strategy: one-step in situ synthesis of self-assembled 3D graphene-based composites with enhanced lithium storage capacity
}

\begin{abstract}
A facile and controllable approach has been developed to synthesize three-dimensional (3D) graphenebased monoliths. Here, as a proof-of-concept experiment, self-assembled 3D CoO/graphene sheets (CoO/ GS) composites with porous structures have been successfully fabricated in an ethanol medium by anestep, in situ growth, solvothermal method. During the process, the in situ nucleation and growth of CoO particles on GS were tuned by the formation of a 3D GS network. In the as-prepared composites, the selfassembled 3D GS network around the $\mathrm{CoO}$ particles can not only provide a 3D conductive matrix, but also buffer the volume changes of $\mathrm{CoO}$ and restrain the aggregation of $\mathrm{CoO}$ particles during charge-discharge cycling. The $\mathrm{CoO}$ particles, which are uniformly anchored into the 3D GS framework, can also act as spacers to effectively avoid the close restacking of GS. Compared to the bare CoO, the 3D CoO/GS composites as Li-ion battery anodes show dramatically improved electrochemical performance, including cycling stability and rate capability, owing to the unique self-assembled 3D structure and the superior synergistic effect between the two components. Such a synthesis strategy can be a promising route to produce diverse 3D graphene-based monoliths in various solvents.
\end{abstract}

\section{Keywords}

one, step, situ, synthesis, solvothermal, self, strategy, assembled, 3d, graphene, composites, enhanced, lithium, storage, capacity

\author{
Disciplines \\ Engineering | Physical Sciences and Mathematics
}

\section{Publication Details}

Ma, J., Wang, J., He, Y., Liao, X., Chen, J., Wang, J., Yuan, T. \& Ma, Z. (2014). A solvothermal strategy: onestep in situ synthesis of self-assembled 3D graphene-based composites with enhanced lithium storage capacity. Journal of Materials Chemistry A, 2 (24), 9200-9207.

\section{Authors}

Jingjing Ma, Jiulin Wang, Yu-Shi He, Xiao Zhen Liao, Jun Chen, Jiazhao Wang, Tao Yuan, and Zi-Feng Ma 
A solvothermal strategy: one-step in situ synthesis of self-assembled 3D graphene-based composites with enhanced lithium storage capacity

Jinjin Ma, ${ }^{\dagger}$ Jiuling Wang, ${ }^{\dagger}$ Yu-Shi He, ${ }^{\dagger, "}$ Jun Chen, ${ }^{\ddagger}$ Jia-Zhao Wang, ${ }^{,}$Tao Yuan, ${ }^{\dagger}$ Xiao-Zhen Liao, ${ }^{\dagger}$ Zi-Feng Ma ${ }^{\dagger}$

'Institute of Electrochemical and Energy Technology, Department of Chemical Engineering, Shanghai Jiao Tong University, Shanghai, 200240, China.

${ }^{\sharp}$ Intelligent Polymer Research Institute, ARC Centre of Excellence for Electromaterials Science, University of Wollongong, Wollongong, NSW, 2522, Australia

${ }^{8}$ Institute for Superconducting and Electronic Materials, University of Wollongong, Wollongong, NSW, 2522, Australia.

${ }^{*}$ Corresponding Author: Yu-Shi He

Tel: +86-21-54744533, Fax: +86-21-54747717

E-mail: ys-he@sjtu.edu.cn

\section{ABSTRACT}

A facile and controllable approach has been developed to synthesize three-dimension (3D) graphene-based monoliths. Here, as a proof-of-concept experiment, self-assembled 3D $\mathrm{CoO} /$ graphene sheets $(\mathrm{CoO} / \mathrm{GS})$ composites with porous structures have been successfully fabricated in ethanol medium by the one-step in situ growth solvothermal method. During the process, the in situ nucleation and growth of $\mathrm{CoO}$ particles on GS were tuned with the formation of 3D GS network. In the as-prepared composites, the self-assembled 3D GS network around $\mathrm{CoO}$ particles can not only provide a 3D conductive matrix, but also buffer the volume change of $\mathrm{CoO}$ and 
restrain the aggregation of $\mathrm{CoO}$ particles. $\mathrm{CoO}$ particles which uniformly anchored on the 3D GS framework can also act as spacers to effectively avoid the close restacking of GS. Comparing to the bare $\mathrm{CoO}$, the $3 \mathrm{D} \mathrm{CoO} / \mathrm{GS}$ composites as Li-ion battery anodes show dramatic improved electrochemical performance including cyclic stability and rate capability owing to the unique self-assembled 3D structure and the superior synergistic effect between the two components. Such a synthesis strategy can be a promising route to produce diverse 3D graphene-based monoliths in various solvents.

Keywords: Solvothermal, in situ, self-assembled, 3D graphene, $\mathrm{CoO}$, anode material

\section{Introduction}

Possessing a combination of remarkable electrical, optical, thermal, and mechanical properties, ${ }^{1,2}$ graphene and graphene-based materials have shown outstanding potentials for a variety of applications, including energy storage, ${ }^{3-5}$ bioapplications, ${ }^{6}$ flexible electronics, ${ }^{7}$ sensors, ${ }^{8}$ etc. The main challenge in the synthesis of the graphene-based composites concentrates on how to effectively inhibit the restacking and agglomeration of GS to fully harness the unique properties of individual graphene sheet and adequately utilize the synergistic effect between GS and other component. The conventional direct high-temperature calcination can easily cause severe agglomeration of the GS. ${ }^{9}$ More recently, many efforts have been made for retaining the structure of graphene network, such as keeping the graphene solvated by vacuum filtration method ${ }^{10}$ and developing a range of 3D graphene-based composites by 
spray drying or hydrothermal technique. ${ }^{11-14}$ Shi et al. ${ }^{13}$ firstly demonstrated that graphene hydrogels composed of a randomly cross-linked 3D GS network can be readily formed when a high concentration of graphene oxide dispersion is hydrothermally reduced without the addition of any reducing agent. Nevertheless, some particular material has to be prepared in specific solvent. By the aid of solvothermal reduction method, some researchers found that GO dispersion can be directly reduced to GS dispersion in some solvents, such as $\mathrm{NMP},{ }^{15}$ ethanol, ${ }^{16-18}$ ethylene glycol, ${ }^{16} \mathrm{~N}, \mathrm{~N}$-dimethylformamide (DMF) ${ }^{19}$ and 1-butanol ${ }^{16}$. However, there are still few reports on the direct preparation of self-assembled 3D graphene-based composites by the simple solvothermal reduction method. Recently, self-assembled 3D graphene-based organogels were prepared by solvothermal reduction of GO dispersion in propylene carbonate $(\mathrm{PC}) .{ }^{20}$ Furthermore, by the solverthermal method and following freeze-drying process, we have also successfully synthesized various 3D GS aerogels in different solvents, including NMP, ethanol, ethylene glycol and DMF (Fig. 1). The obtained 3D GS aerogels can maintain the porous structure of 3D GS organogels via freeze-drying treatment, while naturally drying process will result in the drastic shrinkage of 3D GS and impair the porous structure.

Transition metal oxides (MOs) have been regarded as promising anode materials for next-generation rechargeable Li-ion batteries (LIBs) with high theoretical capacity $\left(>600 \mathrm{mAh} \mathrm{g}^{-1}\right)$ compared to that of conventional graphite $\left(372 \mathrm{mAh} \mathrm{g}^{-1}\right){ }^{21}$ 
Unfortunately, the poor electrochemical performance of MOs, especially cycle stability and rate capability, has hindered their practical application. It can be attributed to their low conductivity and drastic volume variation during the Li ions insertion/extraction process which could cause electrode pulverization and loss of electrical continuity. ${ }^{18}$ Flexible GS-wrapped MOs have been attractive options to alleviate the problems. ${ }^{22,23}$ However, the obvious capacity degradation associated with the aggregation and pulverization of MOs particles still exists due to the loose contact between GS and the MOs particles.

Here, as a proof-of-concept experiment, we demonstrate a one-step in situ growth solvothermal method in ethanol medium to prepare 3D self-assembled CoO/GS composites. The in situ controlled growth of $\mathrm{CoO}$ particles on the surface of GS can effectively avoid the occurrence of concentration gradient during initial reaction stage and form a more homogeneous composite and stronger interaction between GS and $\mathrm{CoO}$ particles with a tighter interface, which can contribute to the interfacial charge transfer and reduce the agglomeration and restacking of GS. ${ }^{24}$ The solvothermal method offers significant advantages in preparation because no reducing agent, no filtration process, no calcination and no protective atmospheres are required. When employed as anode for LIBs, $\mathrm{CoO} / \mathrm{GS}$ delivers superior cycling performance with stably reversible capacity of about $405 \mathrm{mAh} \mathrm{g}^{-1}$ at a high current of $6400 \mathrm{~mA} \mathrm{~g}^{-1}$. Above all, such a facile solvothermal strategy can be applicable for the synthesis of 3D graphene-based monoliths in different solvents. 


\section{Experimental details}

Synthesis of 3D CoO/GS. Graphite oxide was synthesized from natural graphite powder (Grade 230, Asbury Carbons) by a modified Hummer's method. ${ }^{25}$ The graphite oxide ( $5 \mathrm{mg}$ ) was exfoliated into $10 \mathrm{~mL} \mathrm{99.9 \%} \mathrm{ethanol} \mathrm{by} \mathrm{sonication} \mathrm{to} \mathrm{form}$ graphene oxide (GO) suspension. Then, a certain amount of $\mathrm{Co}\left(\mathrm{CH}_{3} \mathrm{COO}\right)_{2} \cdot 4 \mathrm{H}_{2} \mathrm{O}$ were added into the above solution in a weight ratio of $\mathrm{Co}\left(\mathrm{CH}_{3} \mathrm{COO}\right)_{2} \cdot 4 \mathrm{H}_{2} \mathrm{O}: \mathrm{GO}$ of $15: 1$, The mixture was sealed into a glass sample vial. Thereafter, the vial was put in Teflon-lined autoclave and maintained at $180{ }^{\circ} \mathrm{C}$ for $12 \mathrm{~h}$. After it was naturally cooled down to room temperature, a black columniform product was obtained and immersed in water overnight to remove the residual ions, designated as $\mathrm{CoO} / \mathrm{GS}$ (15). 3D CoO/GS (10) and CoO/GS (20) were synthesized by the same method, except that the weight ratio of $\mathrm{Co}\left(\mathrm{CH}_{3} \mathrm{COO}\right)_{2} \cdot 4 \mathrm{H}_{2} \mathrm{O}: \mathrm{GO}$ were $10: 1$ and $20: 1$ respectively. Pure $\mathrm{CoO}$ and GS samples were also prepared by the same procedure for comparison. The all as-prepared samples were freeze-dried for the following tests.

Structural and morphological characterization. X-ray diffraction (XRD) measurements were carried out using a Rigaku D/MAX-2200/PC X-ray diffractometer at $40 \mathrm{kV}$ and $20 \mathrm{~mA}$, with a $\mathrm{Cu} \mathrm{K \alpha}$ radiation source. Raman spectroscopy was used to identify the surface characteristics of the samples using a BRUKER optic SENTERRA (R-200L) Raman spectrometer using a laser with a 
wavelength of $523 \mathrm{~nm}$ at room temperature. FT-IR measurement was carried out on a Perkin- Elmer 936 infrared spectrophotometer from $\mathrm{KBr}$ pellets in the range of 500$4000 \mathrm{~cm}^{-1}$. X-ray photoelectron spectrometer (XPS, Kratos Axis Ultra DLD) was utilized to analyze the surface chemistries of the samples. Thermogravimetric analysis (TGA) was performed using a STA 449F3 analyzer (NETZSCH Co., Germany) to evaluate the residual level of $\mathrm{CoO} / \mathrm{GS}$ composite after calcination. The morphology and microstructure of the samples were monitored using a FEI Nova SEM 230 ultra-high resolution Field Emission Scanning Electron Microscopy (FESEM) equipped with energy dispersive X-ray spectroscopy (EDS, INCA X-Max 80, Oxford Instruments), Transmission Election Microscopy (TEM)/Scanning Transmission Electron Microscopy (STEM) (JEM-2100F, JEOL Ltd., Japan) was operated at 200 $\mathrm{kV}$ equipped with EDS (INCA-IET200, Oxford Instruments). Porosity and BET surface areas for the samples were measured using a nitrogen sorption instrument (Micromeritics, ASAP2020).

Electrochemical measurements. The $\mathrm{CoO} / \mathrm{GS}$ hybrids were dried at $80{ }^{\circ} \mathrm{C}$ for $3 \mathrm{~h}$ under Ar atmosphere. Then, $75 \mathrm{wt} \%$ active material (CoO/GS hybrids), $15 \mathrm{wt} \%$ acetylene black (Super-P), and $10 \mathrm{wt} \%$ polyvinylidene fluoride (PVDF) binder were mixed into N-methyl-2-pyrrolidinone (NMP). The obtained slurry was coated onto $\mathrm{Cu}$ foil disks to form working electrode, the electrode was dried, cut to $\Phi 14 \mathrm{~mm}$ sheets, pressed at $3 \mathrm{MPa}$, and finally dried at $80{ }^{\circ} \mathrm{C}$ in vacuum for $4 \mathrm{~h}$ to remove the solvent. The active materials loaded on the electrode were about $1.5 \mathrm{mg} \cdot \mathrm{cm}^{-2}$. 
CR2016 coin cells were assembled in an argon-filled glove box with lithium metal as counter electrode and UP3025 separator (provided by UBE Industries, Ltd., Japan). The electrolyte contained $1 \mathrm{M} \mathrm{LiPF}_{6}$ in dimethyl carbonate (DMC) and ethylene carbonate (EC) mixed solvent of 1:1 (LP30 from EM Industries, Inc.). Charge-discharge cycles of the half-cells were evaluated between 0.005 and $3 \mathrm{~V}$ vs $\mathrm{Li}+/ \mathrm{Li}$ at room temperature using LAND CT2001A model battery test system (Wuhan Jinnuo Electronics, Ltd.) under constant current condition. The charge-discharge capacities were calculated according to the weight of $\mathrm{CoO} / \mathrm{GS}$ material in the electrode. The AC electrochemical impedance spectra (EIS) tests of the cells were measured by a Solartron FRA 1260 frequency responses analyzer combined with a Solartron SI 1287 Electrochemical Interface with an amplitude of $10 \mathrm{mV}$ over a frequency range from $100 \mathrm{kHz}$ to $0.1 \mathrm{~Hz}$. Cyclic voltammetry (CV) measurements were carried out by using a $\mathrm{CHI}$ instrument (CHI 660) at a scanning rate of $0.5 \mathrm{mV}$ $\mathrm{S}^{-1}$

\section{Results and discussion}

As shown in Fig. 2, the overall formation process of self-assembled 3D CoO/GS composite involves the following steps. Firstly, $\mathrm{Co}\left(\mathrm{CH}_{3} \mathrm{COO}\right)_{2} \cdot 4 \mathrm{H}_{2} \mathrm{O}$ is sonicated with the wetted GO in ethanol to form uniform and opaque dispersion (Fig. 2a). At this place, the functional groups on the surface of GO sheets with negative charge could bind with $\mathrm{Co}^{2+}$ ions by electrostatic interactions and act as anchor sites. After that, the above mixture was solvothermal self-assembled at $180{ }^{\circ} \mathrm{C}$ for $12 \mathrm{~h}$, thus 
forming a black ethanol gel of 3D CoO/GS (Fig. 2b). During the solvothermal reaction, $\mathrm{Co}^{2+}$ ions adsorbed on $\mathrm{GO}$ sheets reacted with $\mathrm{OH}^{-}$ions to form $\mathrm{CoO}$ crystals under high temperature and pressure, ${ }^{26}$ while GO was simultaneously reduced and self-assembled to form 3D GS network. The as-obtained 3D CoO/GS ethanol gel was freeze-dried for maintaining the 3D monolithic network to obtain 3D CoO/GS aerogel (Fig. 2c). It is noteworthy that the shape of the 3D product depends on the geometry of the autoclave.

The XRD patterns of $\mathrm{CoO} / \mathrm{GS}(15)$ and bare $\mathrm{CoO}$ are shown in Fig. 3a. For the $\mathrm{CoO} / \mathrm{GS}$ (15) composite and bare $\mathrm{CoO}$, The major diffraction peaks at $2 \theta$ values of 36.5 (111), 42.4 (200), 61.5 (220), 73.7 (311), and 77.5 (222) match well with those of the standard cubic $\mathrm{CoO}$ (JCPDS 43-1004). No obvious characteristic of GO at about $11^{\circ}$ (Fig. $\mathrm{S} 1 \dagger$ ) is observed in the 3D CoO/GS (15) composite, which suggests that GO was reduced to GS during the solvothermal process. Moreover, the characteristic (002) stacking peak of graphene at $22-28^{\circ}$ (Fig. S1 $\dagger$ ) is also absent here, indicating that the graphene sheets are evenly dispersed without obvious stacking and successfully covered with well-crystallized $\mathrm{CoO}$. The similar phenomenon was also observed in the previous reports about the graphene-based hybrids. ${ }^{12,27}$

Fig. $3 \mathrm{~b}$ exhibits the Raman spectra of $\mathrm{CoO} / \mathrm{GS}$ (15), pristine GS and bare CoO. In the $\mathrm{CoO} / \mathrm{GS}(15)$, the peaks below $1000 \mathrm{~cm}^{-1}$ are consistent with the characteristic peaks of bare $\mathrm{CoO}$. Characteristic peaks for carbon materials include the disordered D band 
at about $1350 \mathrm{~cm}^{-1}$, the graphitic $\mathrm{G}$ band at about $1590 \mathrm{~cm}^{-1}$, the 2D band at about $2700 \mathrm{~cm}^{-1}$ and $\mathrm{D}^{*}$ at about $2900 \mathrm{~cm}^{-1} .{ }^{28}$ The distinct $\mathrm{D}$ band peak and the relative small 2D peak indicate a lot of dangling bonds, defects, and disordered structure present. ${ }^{29}$ These defects could act as nucleation sites for $\mathrm{CoO}$ particles growing and allow uniform formation of $\mathrm{CoO}$ particles in 3D GS network. ${ }^{30}$ Furthermore, in comparison with the pristine GS, the shift of the peaks can be clearly found for both D and G bands in the CoO/GS (15) composite, indicating a significant charge transfer between the 3D GS and $\mathrm{CoO}$ particles. The charge transfer between GS and $\mathrm{CoO}$ can effectively improve the electrochemical performance of the CoO/GS composite. ${ }^{31}$

The FT-IR spectrum of the CoO/GS (15) composite shows peaks at 550 and $665 \mathrm{~cm}^{-1}$ that can be assigned to the vibrations of Co-O (Fig. S2 $\dagger$ ). ${ }^{23}$ The peak located at 1224 $\mathrm{cm}^{-1}$ is derived from epoxy $(\mathrm{C}-\mathrm{O}-\mathrm{C})$ groups, while the peak presented at $1570 \mathrm{~cm}^{-1}$ corresponds to the stretching vibration of $\mathrm{C}=\mathrm{C} .^{32}$

XPS analysis was used to further characterize the surface chemical composition of CoO/GS (15) composite. Full XPS spectrum (Fig. 4a) firstly indicates the presence of only $\mathrm{Co}$, $\mathrm{O}$ and $\mathrm{C}$ elements in the composite. In Fig. 4b, four different peaks which are corresponding to carbon $\mathrm{sp}^{2}\left(C_{g}, \sim 285 \mathrm{eV}\right)$, epoxy/hydroxyl groups $(\mathrm{C}-\mathrm{O}, \sim 286.5$ $\mathrm{eV})$, carbonyl group $(\mathrm{C}=\mathrm{O}, \sim 287.8 \mathrm{eV})$ and carboxyl group $(\mathrm{O}-\mathrm{C}=\mathrm{O}, \sim 289.5 \mathrm{eV})$, respectively, are detected. ${ }^{33}$ The fraction of carbon-carbon bonding is about $71.9 \%$, which is similar to the previously reported result. ${ }^{12}$ It can be attributed to the efficient 
removal of oxygen functional groups from the GO by the solvothermal reaction and demonstrate the formation of graphene. The high-resolution Co $2 p$ spectrum of the $3 \mathrm{D}$ composite is shown in Fig. 3c. The peaks at 782.1 and $797.6 \mathrm{eV}$ with a $15.5 \mathrm{eV}$ peak-to-peak separation correspond to the binding energy of Co $2 p 3 / 2$ and $2 p \quad 1 / 2$ of $\mathrm{CoO}$, respectively, while two weak peaks at 787.4 and $803.8 \mathrm{eV}$ should be the shake-up satellite peaks of above two main peaks, indicating the presence of Co (II ). ${ }^{27}$ The aforementioned XRD, Raman, FTIR and XPS characterizations confirm the successful preparation of $\mathrm{CoO} / \mathrm{GS}$ composite.

FESEM images in Fig. 5 show the surface morphologies of $(a, b)$ bare $\mathrm{CoO}(\mathrm{c}, \mathrm{d})$ pristine GS and (e,f) CoO/GS (15). The SEM images of the bare CoO (Fig. 5a and 5b) display irregular particles about $200-500 \mathrm{~nm}$ in diameter with rough surfaces. From Fig. 5c and 5d, it can be clearly observed that the pure GS exhibits an easily recognizable and interconnected 3D porous framework with the pore sizes ranging from submicrometer to several micrometres. The formation of the self-assembled 3D GS framework is derived from the regional overlapping and coalescing of flexible GS through $\pi-\pi$ stacking interactions during solvothermal treatment. ${ }^{13,34}$ As shown in Fig. 5e and 5f, CoO/GS (15) still possesses a fully interconnected macroporous architecture, which is similar to pristine GS. But it is evident that the pore sizes of the 3D GS network in the composite became much larger due to the introduction of $\mathrm{CoO}$ particles. The sphere-like $\mathrm{CoO}$ particles with a mean diameter of about $350 \mathrm{~nm}$ in the CoO/GS (15) composite are uniformly and closely anchored on the 3D GS network, 
suggesting effective assembly between the $\mathrm{CoO}$ particles and GS during solvothermal treatment. And the surface morphology of the $\mathrm{CoO}$ particles in the composite is totally different from the bare $\mathrm{CoO}$, which is ascribed that the oxygen-containing functional groups (hydroxyl, carboxyl and epoxy etc.) on GO surface/edge could improve the crystallization of $\mathrm{CoO}$ and also act as anchor sites for in situ formation of $\mathrm{CoO}$ particles. ${ }^{35}$ It is notable that most of $\mathrm{CoO}$ particles are clearly encapsulated in the GS matrix and the graphene shells present a typical crumpled and rippled morphology (Fig. 5f). It has been reported that the crumpled GS encapsulation not only enhances interface contact, but also suppresses the aggregation of particles and provides elastic void spaces to accommodate the strain and stress of the volume change of electrode materials during cycling. ${ }^{36}$

From the result of the EDS mappings in a relatively large area (about $4 \mu \mathrm{m} \times 4 \mu \mathrm{m}$ ) for carbon, oxygen and cobalt elements (Fig. S3†), it can be found that the $\mathrm{CoO}$ particles are uniformly distributed in the 3D GS network. The graphene layer on some $\mathrm{CoO}$ surface may be too thin to be visible in the SEM image. So combined with EDS analysis (Fig. 6f), a STEM image (Fig. 6a) and corresponding elemental mapping images (Fig. 6c-e) of the small region indicated in Fig. 6b provide additional evidence to further confirm the homogeneous distribution of carbon, oxygen and cobalt on the surface of individual $\mathrm{CoO}$ particle.

Microstructures of various samples were further characterized by TEM and high-resolution TEM (HRTEM) (Fig. 7). A typical TEM image of the prepared bare 
CoO sample can be observed in Fig. 6a. The morphology of the $\mathrm{CoO}$ particles with rough surfaces is irregular, which is identical with the SEM observation. The corresponding selected-area electron diffraction (SAED) pattern (inset in Fig. 7a) shows clear diffraction spots, demonstrating the single-crystal-like nature. In Fig. S4†, the pure GS exhibits a transparent feature with a wrinkled structure. Fig. $7 \mathrm{~b}$ and $7 \mathrm{c}$ show the TEM images of the $\mathrm{CoO} / \mathrm{GS}$ (15) composite. As expected, the $\mathrm{CoO}$ particles are well wrapped by the GS and are distributed homogeneously in the network. The inset in Fig. 7b depicts the corresponding SAED pattern of the CoO/GS (15) hybrid. There are two sets of diffraction patterns that belong to the CoO and GS, respectively. Note that the weak diffraction rings from GS are obvious due to multilayer graphene and the diffraction spots for $\mathrm{CoO}$ demonstrate the single-crystal in nature of the $\mathrm{CoO}$ in CoO/GS (15) composite. ${ }^{23,26}$ The HRTEM image of the CoO/GS (15) composite in Fig. $7 \mathrm{~d}$ indicates that the $\mathrm{CoO}$ particle is encapsulated by the multiple overlapping GS. And the regular lattice fringes show a spacing of $0.24 \mathrm{~nm}$, which can be assigned to the (111) plane of $\mathrm{CoO}^{23}$ In addition, the graphene content in the $\mathrm{CoO} / \mathrm{GS}$ composite could significantly influence the morphology of the product. Based on the TEM images of CoO/GS (10) (Fig. S5a $\dagger$ ), CoO/GS (15) (Fig. 7b) and CoO/GS (20) (Fig. $\mathrm{S} 5 \mathrm{~b} \dagger)$, it can be found that, with the decrease of the graphene content, the sizes of the $\mathrm{CoO}$ particles gradually become larger. This result illustrates that the size of as-synthesized material particles in 3D GS network is controllable by the in situ growth solvothermal process. 
For quantifying the amount of graphene in the $\mathrm{CoO} / \mathrm{GS}$ composites, TGA was carried out at a heating rate of $10{ }^{\circ} \mathrm{C} \min ^{-1}$ from $40{ }^{\circ} \mathrm{C}$ to $700{ }^{\circ} \mathrm{C}$ in air. In Fig. S6†, the weight loss at the temperature below $100{ }^{\circ} \mathrm{C}$ is attributed to the evaporation of residual water in the samples and the rapid mass loss between 220 and $350{ }^{\circ} \mathrm{C}$ could be due to the combustion of the graphene. ${ }^{23,37}$ Therefore, the weight fractions of graphene in the $\mathrm{CoO} / \mathrm{GS}(10), \mathrm{CoO} / \mathrm{GS}(15)$ and $\mathrm{CoO} / \mathrm{GS}$ (20) were determined to be about $17.78 \%, 12.41 \%$ and $8.44 \%$, respectively.

Fig. 8 shows the electrochemical performance of the CoO/GS (15) composite. To identify the mechanism of the electrochemical reactions, the $\mathrm{CV}$ profiles of the $\mathrm{CoO} / \mathrm{GS}$ (15) for the first, second and teenth cycles were measured at a scan rate of $0.5 \mathrm{mV} \mathrm{s}^{-1}$ as shown in Fig. 8a. In the cathodic polarization process of the first cycle for $\mathrm{CoO} / \mathrm{GS}$ (15), two peaks, one obvious and one inconspicuous, were observed at about 0.28 and $0.98 \mathrm{~V}$, which were ascribed to the $\mathrm{Li}$ insertion into $\mathrm{CoO} / \mathrm{GS}$ composite and the formation of solid electrolyte interphase (SEI) film. ${ }^{37}$ Apparently, the reaction indicates the occurrence of some irreversible processes in the first cycle. Meanwhile, one broadened peak was recorded at about $2.2 \mathrm{~V}$ in the anodic process, corresponding to the reversible oxidation of cobalt to cobalt oxide. ${ }^{38}$ During the subsequent cycles, the cathodic peak potential transfers to higher voltage potentials and the CV curves show good reproducibility, suggesting the good reversible reaction. For $\mathrm{CoO} / \mathrm{GS}$ electrode, the electrochemical reversible reaction mechanism of Li with $\mathrm{CoO}$ and carbon in lithium ion battery could be described as follows: ${ }^{39}$

$$
\mathrm{CoO}+2 \mathrm{Li}^{+}+2 e^{-} \Leftrightarrow 2 \mathrm{Co}+\mathrm{Li}_{2} \mathrm{O}
$$




$$
6 \mathrm{C}+\mathrm{Li}^{+}+e^{-} \Leftrightarrow \mathrm{LiC}_{6}
$$

Fig. $8 \mathrm{~b}$ show the charge (delithiation) and discharge (lithiation) voltage profiles for the $\mathrm{CoO} / \mathrm{GS}$ (15) composite at a current density of $200 \mathrm{~mA} \mathrm{~g}^{-1}$ at room temperature. In the first discharge cycle, an extended potential plateau at around $0.8 \mathrm{~V}$ demonstrates the conversion reaction and the formation of SEI film, which is consistent with the $\mathrm{CV}$ results. The first discharge capacities $\left(1413 \mathrm{mAh} \mathrm{g}^{-1}\right)$ of the composite is much higher than the theoretical capacity of $\mathrm{CoO}\left(716 \mathrm{mAh} \mathrm{g}^{-1}\right)$, which may derive from the decomposition of electrolyte, the formation of the SEI layer and the reduction of oxygenated functional groups on the surface of graphene in the $\mathrm{CoO} / \mathrm{GS}$ (15) composite. $^{22,23}$ Furthermore, it was reported that graphene can also contribute additional lithium storage capacity apart from the intrinsic theoretical capacity, which is due to significant disorder/defects of graphene. ${ }^{40}$ The coulombic efficiency (CE) of the $\mathrm{CoO} / \mathrm{GS}$ (15) composite is around $77 \%$ at the first cycle and $\mathrm{CE}$ rises to more than $98 \%$ after 1 cycle. For the composite, it is notable that no obvious capacity loss was observed after 2 cycles and the electrode could still maintain a reversible capacity of approximately $960 \mathrm{mAh} \mathrm{g}^{-1}$ after 10 cycles.

To evaluate the electrode kinetics of bare $\mathrm{CoO}, \mathrm{CoO} / \mathrm{GS}(10), \mathrm{CoO} / \mathrm{GS}$ (15) and $\mathrm{CoO} / \mathrm{GS}(20)$, the rate capability of the samples is shown in Fig. 8c. It is clearly seen that the $\mathrm{CoO} / \mathrm{GS}$ composites, especially the $\mathrm{CoO} / \mathrm{GS}$ (15) composite with $12.3 \%$ graphene, illustrate much better rate performance comparing to the reference bare $\mathrm{CoO}$ and other $\mathrm{CoO} @$ GS composites. When the charge/discharge current density 
increases to $1600 \mathrm{~mA} \mathrm{~g}^{-1}$, the reversible capacity of the CoO/GS (15) composite still keeps a stable value above $730 \mathrm{mAh} \mathrm{g}^{-1}$. In the contrast, at this high rate, the bare $\mathrm{CoO}$ can only deliver an average reversible capacity of $c a .270 \mathrm{mAh} \mathrm{g} \mathrm{g}^{-1}$. When the current density is returned to $200 \mathrm{~mA} \mathrm{~g}^{-1}$, the $\mathrm{CoO} / \mathrm{GS}$ (15) electrode can still release a high reversible capacity (1036 $\mathrm{mAh}^{-1}, 95.5 \%$ of the initial reversible capacity) after the 50th cycle, indicating good reversibility.

The discharge-charge cycling performance of $\mathrm{CoO} / \mathrm{GS}$ (15) at high current densities was further evaluated in Fig. 8d. All cells were cycled at a current density of $200 \mathrm{~mA}$ $\mathrm{g}^{-1}$ for the initial two cycles before each test. The composite exhibited a good cycling performance at high current densities. After 50 cycles, stable reversible capacities of about 706, 503 and $405 \mathrm{mAh} \mathrm{g}^{-1}$ can still be retained at current densities of 1600 , 4800 and $6400 \mathrm{~mA} \mathrm{~g}^{-1}$, respectively. Moreover, the high-rate cycling performance of CoO@GS (15) composite is much better than that of bare $\mathrm{CoO}$, whose reversible capacity is only $211 \mathrm{mAh} \mathrm{g}^{-1}$ after the same 50 cycles at a current density of $1600 \mathrm{~mA}$ $\mathrm{g}^{-1}$

Evidently, the $\mathrm{CoO} / \mathrm{GS}$ composites show significantly enhanced cycling and rate performance perhaps benefited from the unique self-assembled 3D structure of GS and simultaneous growth of $\mathrm{CoO}$ particles anchored on 3D GS framework during the solvothermal route, which can ensure the integrity of the electrodes over many discharge/charge cycles. This can be confirmed by examining the microstructure 
variation of the bare $\mathrm{CoO}$ and the $\mathrm{CoO} / \mathrm{GS}$ (15) composite after 50 cycles at a charge/discharge current density of $1600 \mathrm{mAh} \mathrm{g}^{-1}$ using TEM (Fig. 9). In Fig. 9a, it is obviously observed that the bare $\mathrm{CoO}$ particles became smaller and cracked after 50 cycles, indicating the pulverization of the particles during cycling that leads to the poor cycling stability of bare $\mathrm{CoO}$ particles. In contrast, in Fig. 9b, the $\mathrm{CoO}$ particle anchored on GS still retained its original morphology and did not further break after 50 cycles. This demonstrates the long-term stability of the as-obtained $\mathrm{CoO} / \mathrm{GS}$ (15) cycling and the pulverization of the $\mathrm{CoO}$ particles is inhibited under the protection of flexible 3D GS network. Additionally, the corresponding SAED results indicate that the $\mathrm{CoO} / \mathrm{GS}$ (15) electrode exhibits better crystallinity than the bare $\mathrm{CoO}$ electrode after 50 cycles,

In order to further clarify the improved electrochemical performance after introducing the graphene into the $\mathrm{CoO} / \mathrm{GS}$ composite, the Nyquist plots of the AC impedance for the bare $\mathrm{CoO}$ and $\mathrm{CoO} / \mathrm{GS}$ (15) composite were measured after 1 and 50 cycles at a charge/discharge current density of $1600 \mathrm{mAh} \mathrm{g}^{-1}$ (Fig. S7 $\dagger$ ). To maintain uniformity, EIS was performed on working electrodes in the fully charged (delithiated) state. The depressed semicircle in the high-medium frequency is related to the capacitance property of the electrode interface. And the inclined line in the low frequency range represents the Warburg impedance, which corresponds to solid-state diffusion of Li ions within the electrodes. ${ }^{29}$ After 1 cycle, the semicircle of the CoO/GS (15) electrode in the high-medium frequency is similar to that of the bare $\mathrm{CoO}$ electrode in 
Fig. S7a $\uparrow$. Nevertheless, after 50 cycles, it is evident that the semicircle for the $\mathrm{CoO} / \mathrm{GS}$ (15) electrode is much smaller than that of the bare $\mathrm{CoO}$ electrode in Fig. $\mathrm{S} 7 \mathrm{~b} \uparrow$, which suggests that the $\mathrm{CoO} / \mathrm{GS}$ (15) electrode possesses lower ohmic and capacitive resistances, which means the improved electronic connection and charge/discharge property of the $\mathrm{CoO}$ electrode could be achieved by effective encapsulation and adherence of highly conductive and flexible GS.

Overall, the improved cycle and rate performance of CoO/GS composites as anode materials for lithium storage may be attributed to the following three aspects. First, almost every individual $\mathrm{CoO}$ particle can be encapsulated into GS and keep intimate contact with GS via the one-step in situ growth solvothermal process. Second, the self-assembled GS in the CoO/GS composites constitutes not only 3D continuous and highly conductive network, but also provides elastic void spaces to buffer the strain and stress of the volume change of $\mathrm{CoO}$ and inhibit the aggregation and pulverization of $\mathrm{CoO}$ particles during cycling, and thus ensures favorable transport kinetics for lithium ions and electrons. Third, $\mathrm{CoO}$ particles are uniformly anchored into the GS matrix as spacers, which can effectively prevent the closely restacking of GS and consequently maintain the large contact area between the electrode and electrolyte.

\section{Conclusions}

In conclusion, a simple but effective and controllable method has been developed to prepare 3D graphene-based monoliths. Here, 3D self-assembled CoO/GS aerogels 
have been successfully fabricated in ethanol medium by the one-step in situ growth solvothermal method and subsequent freeze-drying process, which can be used as anode materials for LIBs. In this way, $\mathrm{CoO}$ particles can in situ nucleate and grow on the surface of GS with the simultaneous formation of 3D GS network. In such a unique macroporous structure, $\mathrm{CoO}$ particles are evenly anchored into the GS matrix. The 3D GS in the composites not only acts as an electronically conductive matrix, but also provides double protection against the aggregation and pulverization of $\mathrm{CoO}$ particles during cycling. And so the synergistic effect between 3D GS and active particles is fully utilized. As a consequence, the as-formed 3D CoO/GS composites show superior cyclic stability and rate capability. The different forms of graphene-based materials can be used in various applications because the outcome is likely to vary with size, morphology and chemical structure. So our study suggests that the size of as-synthesized material particles in 3D GS architecture can be further controlled for other applications by changing concentration of reactants, reaction temperature and reaction time. Most importantly, this study is also a base for developing various 3D graphene-based monoliths in different solvents by the one-step solvothermal strategy for wide applications in near future.

\section{Acknowledgements}

We are grateful for the financial support of this work by the National Basic Research Program of China (2014CB239700), the Natural Science Foundation of China (21336003, 21006063, 21073120, 51272156).

\section{Notes and references}


(1) K. S. Novoselov,A. K. Geim,S. V. Morozov,D. Jiang,Y. Zhang,S. V. Dubonos,I. V. Grigorieva,A. A. Firsov Electric Field Effect in Atomically Thin Carbon Films. Science 2004, 306, 666-9.

(2) C. N. Rao,A. K. Sood,K. S. Subrahmanyam,A. Govindaraj Graphene: The New Two-Dimensional Nanomaterial. Angew Chem Int Ed Engl 2009, 48, 7752-77.

(3) H. Jiang,P. S. Lee,C. Li 3d Carbon Based Nanostructures for Advanced Supercapacitors. Energy \& Environmental Science 2013, 6, 41.

(4) S. Yang,X. Feng,S. Ivanovici,K. Mullen Fabrication of Graphene-Encapsulated Oxide Nanoparticles: Towards High-Performance Anode Materials for Lithium Storage. Angew Chem Int Ed Engl 2010, 49, 8408-11.

(5) Y.-S. He,D.-W. Bai,X. Yang,J. Chen,X.-Z. Liao,Z.-F. Ma A Co(Oh)2-Graphene Nanosheets Composite as a High Performance Anode Material for Rechargeable Lithium Batteries. Electrochemistry Communications 2010, 12, 570-573.

(6) J. Lu,Y.S. He,C. Cheng,Y. Wang,L. Qiu,D. Li,D. Zou Self-Supporting Graphene Hydrogel Film as an Experimental Platform to Evaluate the Potential of Graphene for Bone Regeneration. Advanced Functional Materials 2013, 23, 3494-3502.

(7) T.-H. Han,Y. Lee,M.-R. Choi,S.-H. Woo,S.-H. Bae,B. H. Hong,J.-H. Ahn,T.-W. Lee Extremely Efficient Flexible Organic Light-Emitting Diodes with Modified Graphene Anode. Nature Photonics 2012, 6, 105-110.

(8) W. Yang,K. R. Ratinac,S. P. Ringer,P. Thordarson,J. J. Gooding,F. Braet Carbon Nanomaterials in Biosensors: Should You Use Nanotubes or Graphene? Angew Chem Int Ed Engl 2010, 49, 2114-38.

(9) Y. Wang,Z. Shi,Y. Huang,Y. Ma,C. Wang,M. Chen,Y. Chen Supercapacitor Devices Based on Graphene Materials. The Journal of Physical Chemistry C 2009, 113, 13103-13107.

(10) X. Yang,J. Zhu,L. Qiu,D. Li Bioinspired Effective Prevention of Restacking in Multilayered Graphene Films: Towards the Next Generation of High-Performance Supercapacitors. Advanced Materials 2011, 23, 2833-8.

(11) Y.-S. He,P. Gao,J. Chen,X. Yang,X.-Z. Liao,J. Yang,Z.-F. Ma A Novel Bath Lily-Like Graphene Sheet-Wrapped Nano-Si Composite as a High Performance Anode Material for Li-lon Batteries. RSC Advances 2011, 1, 958.

(12) G.-W. Zhou,J. Wang,P. Gao,X. Yang,Y.-S. He,X.-Z. Liao,J. Yang,Z.-F. Ma Facile Spray Drying Route for the Three-Dimensional Graphene-Encapsulated Fe2o3nanoparticles for Lithium Ion Battery Anodes. Industrial \& Engineering Chemistry Research 2013, 52, 1197-1204.

(13) Y. Xu,K. Sheng,C. Li,G. Shi Self-Assembled Graphene Hydrogel Via a One-Step Hydrothermal Process. ACS Nano 2010, 4, 4324-30.

(14) Z. S. Wu,Y. Sun,Y. Z. Tan,S. Yang,X. Feng,K. Mullen Three-Dimensional Graphene-Based Macro- and Mesoporous Frameworks for High-Performance Electrochemical Capacitive Energy Storage. I Am Chem Soc 2012, 134, 19532-5.

(15) S. Dubin,S. Gilje,K. Wang,V. C. Tung,K. Cha,A. S. Hall,J. Farrar,R. Varshneya,Y. Yang,R. B. Kaner A One-Step, Solvothermal Reduction Method for Producing Reduced Graphene Oxide Dispersions in Organic Solvents. ACS Nano 2010, 4, 3845-52.

(16) C. Nethravathi,M. Rajamathi Chemically Modified Graphene Sheets Produced by the Solvothermal Reduction of Colloidal Dispersions of Graphite Oxide. Carbon 2008, 46, 1994-1998.

(17) S. P. Lim, N. M. Huang,H. N. Lim Solvothermal Synthesis of Sno2/Graphene Nanocomposites for Supercapacitor Application. Ceramics International 2013, 39, 6647-6655.

(18) J. Zhu,T. Zhu,X. Zhou,Y. Zhang,X. W. Lou,X. Chen,H. Zhang,H. H. Hng,Q. Yan Facile Synthesis of 
Metal Oxide/Reduced Graphene Oxide Hybrids with High Lithium Storage Capacity and Stable Cyclability. Nanoscale 2011, 3, 1084-9.

(19) F. He,N. Niu,F. Qu,S. Wei,Y. Chen,S. Gai,P. Gao,Y. Wang,P. Yang Synthesis of Three-Dimensional Reduced Graphene Oxide Layer Supported Cobalt Nanocrystals and Their High Catalytic Activity in F-T Co2 Hydrogenation. Nanoscale 2013, 5, 8507-16.

(20) Q. Zhou,J. Gao,C. Li,J. Chen,G. Shi Composite Organogels of Graphene and Activated Carbon for Electrochemical Capacitors. Journal of Materials Chemistry A 2013, 1, 9196.

(21) P. Poizot,S. Laruelle,S. Grugeon,L. Dupont,J. M. Tarascon Nano-Sized Transition-Metal Oxides as Negative-Electrode Materials for Lithium-Ion Batteries. Nature 2000, 407, 496-9.

(22) M. Zhang,M. Jia,Y. Jin,X. Shi Synthesis and Electrochemical Performance of Coo/Graphene Nanocomposite as Anode for Lithium Ion Batteries. Applied Surface Science 2012, 263, 573-578.

(23) Y. Qi,H. Zhang,N. Du,D. Yang Highly Loaded Coo/Graphene Nanocomposites as Lithium-lon Anodes with Superior Reversible Capacity. Journal of Materials Chemistry A 2013, 1, 2337.

(24) J. Zhou,G. Tian,Y. Chen,X. Meng,Y. Shi,X. Cao,K. Pan,H. Fu In Situ Controlled Growth of Znin2s4 Nanosheets on Reduced Graphene Oxide for Enhanced Photocatalytic Hydrogen Production Performance. Chem Commun (Camb) 2013, 49, 2237-9.

(25) D. Li,M. B. Muller,S. Gilje,R. B. Kaner,G. G. Wallace Processable Aqueous Dispersions of Graphene Nanosheets. Nat Nanotechnol 2008, 3, 101-5.

(26) Y. Ye,F. Yuan,S. Li Synthesis of Coo Nanoparticles by Esterification Reaction under Solvothermal Conditions. Materials Letters 2006, 60, 3175-3178.

(27) Y. Sun,X. Hu,W. Luo,Y. Huang Ultrathin Coo/Graphene Hybrid Nanosheets: A Highly Stable Anode Material for Lithium-Ion Batteries. The Journal of Physical Chemistry C 2012, 116, 20794-20799.

(28) A. C. Ferrari,J. C. Meyer,V. Scardaci,C. Casiraghi,M. Lazzeri,F. Mauri,S. Piscanec,D. Jiang,K. S. Novoselov,S. Roth,A. K. Geim Raman Spectrum of Graphene and Graphene Layers. Physical Review Letters 2006, 97.

(29) L. Yin,J. Wang,F. Lin,J. Yang,Y. Nuli Polyacrylonitrile/Graphene Composite as a Precursor to a Sulfur-Based Cathode Material for High-Rate Rechargeable Li-S Batteries. Energy \& Environmental Science 2012, 5, 6966.

(30) K. Evanoff,A. Magasinski,J. Yang,G. Yushin Nanosilicon-Coated Graphene Granules as Anodes for Li-Ion Batteries. Advanced Energy Materials 2011, 1, 495-498.

(31) W. Chen,S. Li,C. Chen,L. Yan Self-Assembly and Embedding of Nanoparticles by in Situ Reduced Graphene for Preparation of a 3d Graphene/Nanoparticle Aerogel. Adv Mater 2011, 23, 5679-83.

(32) J. Zhou,H. Song,L. Ma,X. Chen Magnetite/Graphene Nanosheet Composites: Interfacial Interaction and Its Impact on the Durable High-Rate Performance in Lithium-Ion Batteries. RSC Advances 2011, 1, 782.

(33) H. Tang,G. J. Ehlert,Y. Lin,H. A. Sodano Highly Efficient Synthesis of Graphene Nanocomposites. Nano Lett 2012, 12, 84-90.

(34) Y. Sun,Q. Wu,G. Shi Supercapacitors Based on Self-Assembled Graphene Organogel. Phys Chem Chem Phys 2011, 13, 17249-54.

(35) J. Yuan,J. Zhu,H. Bi,X. Meng,S. Liang,L. Zhang,X. Wang Graphene-Based 3d Composite Hydrogel by Anchoring Co3o4 Nanoparticles with Enhanced Electrochemical Properties. Phys Chem Chem Phys 2013, 15, 12940-5.

(36) Z. S. Wu,S. Yang,Y. Sun,K. Parvez,X. Feng,K. Mullen 3d Nitrogen-Doped Graphene Aerogel-Supported Fe304 Nanoparticles as Efficient Electrocatalysts for the Oxygen Reduction 
Reaction. J Am Chem Soc 2012, 134, 9082-5.

(37) C. Peng,B. Chen,Y. Qin,S. Yang,C. Li,Y. Zuo,S. Liu,J. Yang Facile Ultrasonic Synthesis of Coo Quantum Dot/Graphene Nanosheet Composites with High Lithium Storage Capacity. ACS Nano 2012, 6, 1074-81.

(38) F. D. Wu,Y. Wang Self-Assembled Echinus-Like Nanostructures of Mesoporous Coo Nanorod@Cnt for Lithium-Ion Batteries. Journal of Materials Chemistry 2011, 21, 6636.

(39) X.-I. Huang,R.-z. Wang,D. Xu,Z.-I. Wang,H.-g. Wang,J.-j. Xu,Z. Wu,Q.-c. Liu,Y. Zhang,X.-b. Zhang Homogeneous Coo on Graphene for Binder-Free and Ultralong-Life Lithium Ion Batteries. Advanced Functional Materials 2013, n/a-n/a.

(40) D. Pan,S. Wang,B. Zhao,M. Wu,H. Zhang,Y. Wang,Z. Jiao Li Storage Properties of Disordered Graphene Nanosheets. Chemistry of Materials 2009, 21, 3136-3142. 
A solvothermal strategy: one-step in situ synthesis of self-assembled 3D graphene-based composites with enhanced lithium storage capacity 


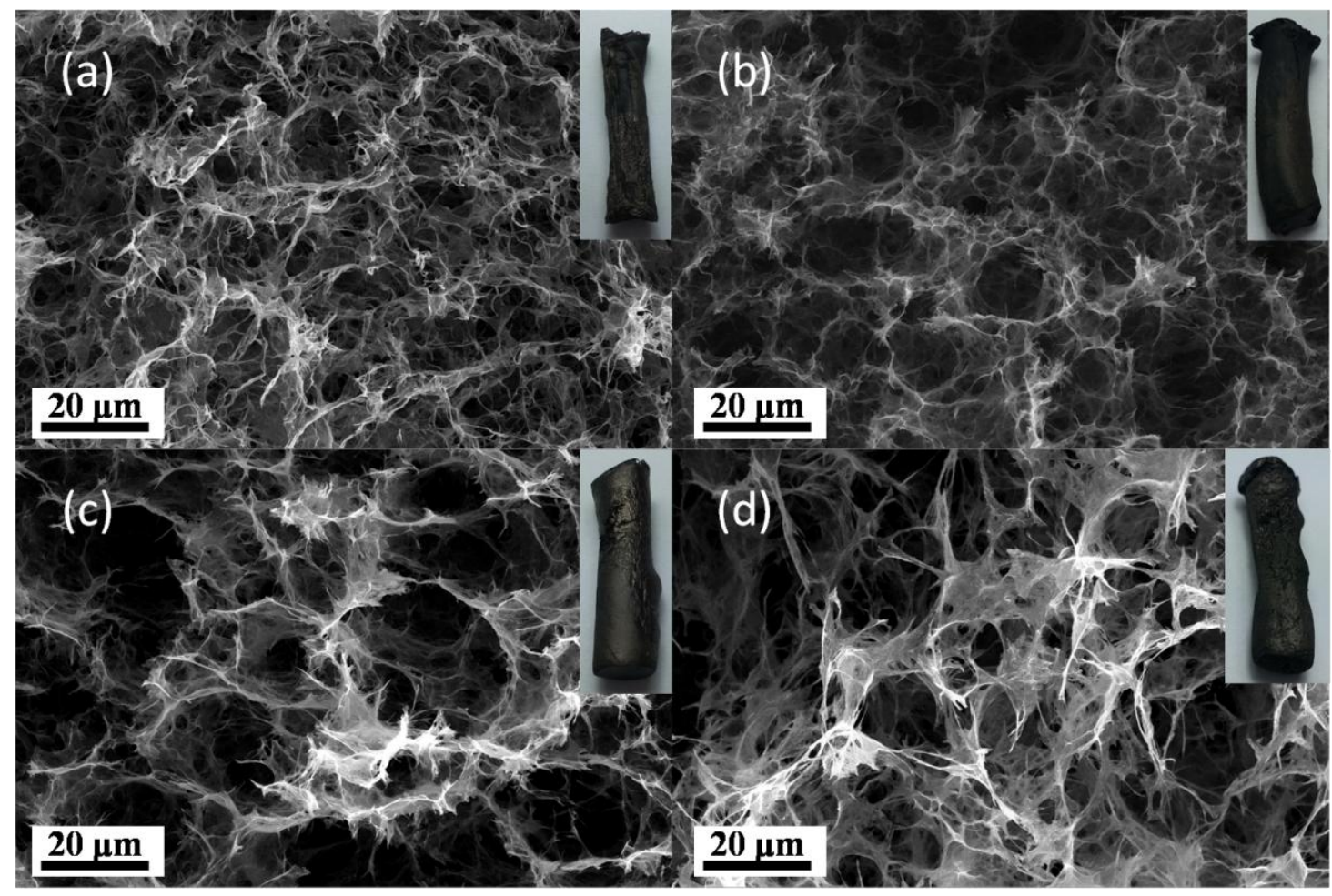

Fig. 1 SEM images of freeze-dried 3D GS aerogels obtained in different solvents (a) NMP, (b) ethanol, (c) ethylene glycol and (d) DMF at $180{ }^{\circ} \mathrm{C}$ for $12 \mathrm{~h}$ in a Teflon-lined autoclave. Insets are the corresponding digital images. GO concentration: $1 \mathrm{mg} \mathrm{mL}^{-1}$. 


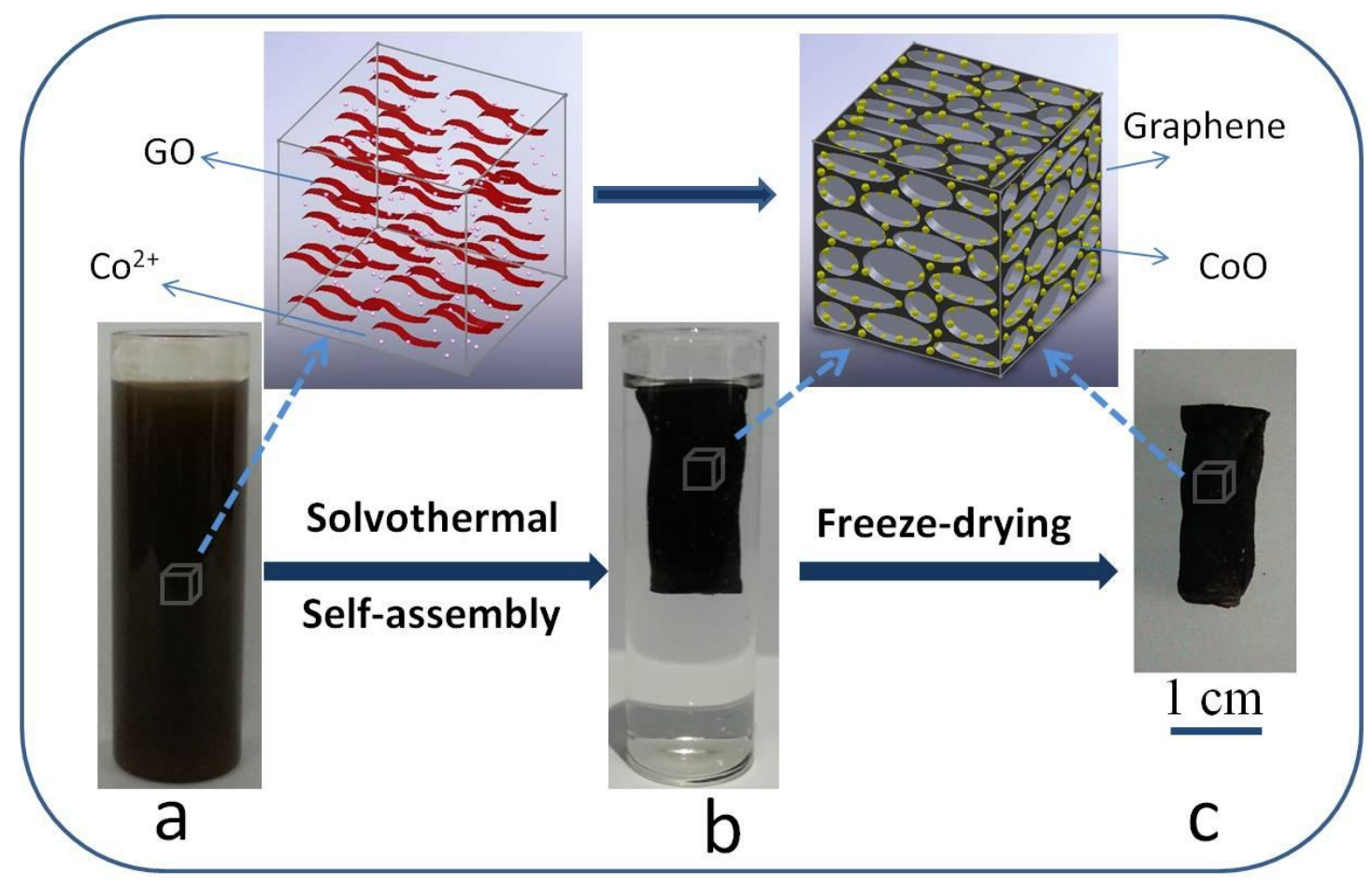

Fig. 2 Formation process of self-assembled 3D CoO/GS composite. a) Uniform and opaque black dispersion of $\mathrm{GO}$ and $\mathrm{Co}\left(\mathrm{CH}_{3} \mathrm{COO}\right)_{2} \cdot 4 \mathrm{H}_{2} \mathrm{O}$ in ethanol. b) 3D CoO/GS hybrid ethanol gel synthesized by solvothermal self-assembly in ethanol. c) 3D CoO/GS hybrid aerogel obtained after freeze-drying. 

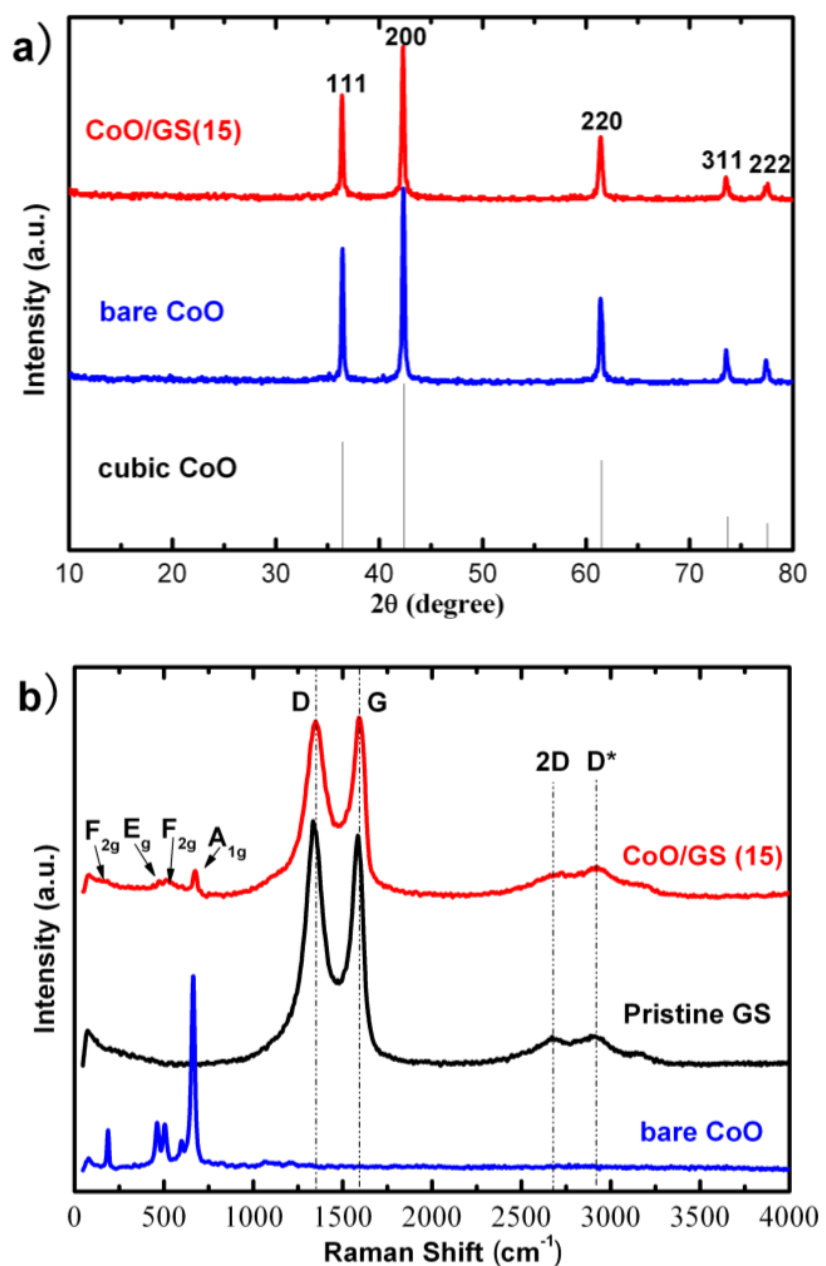

Fig. 3 (a) XRD patterns for the CoO/GS (15) and bare CoO. (b) Raman spectra for the CoO/GS (15), pristine GS and bare $\mathrm{CoO}$ obtained using the standard procedure. 

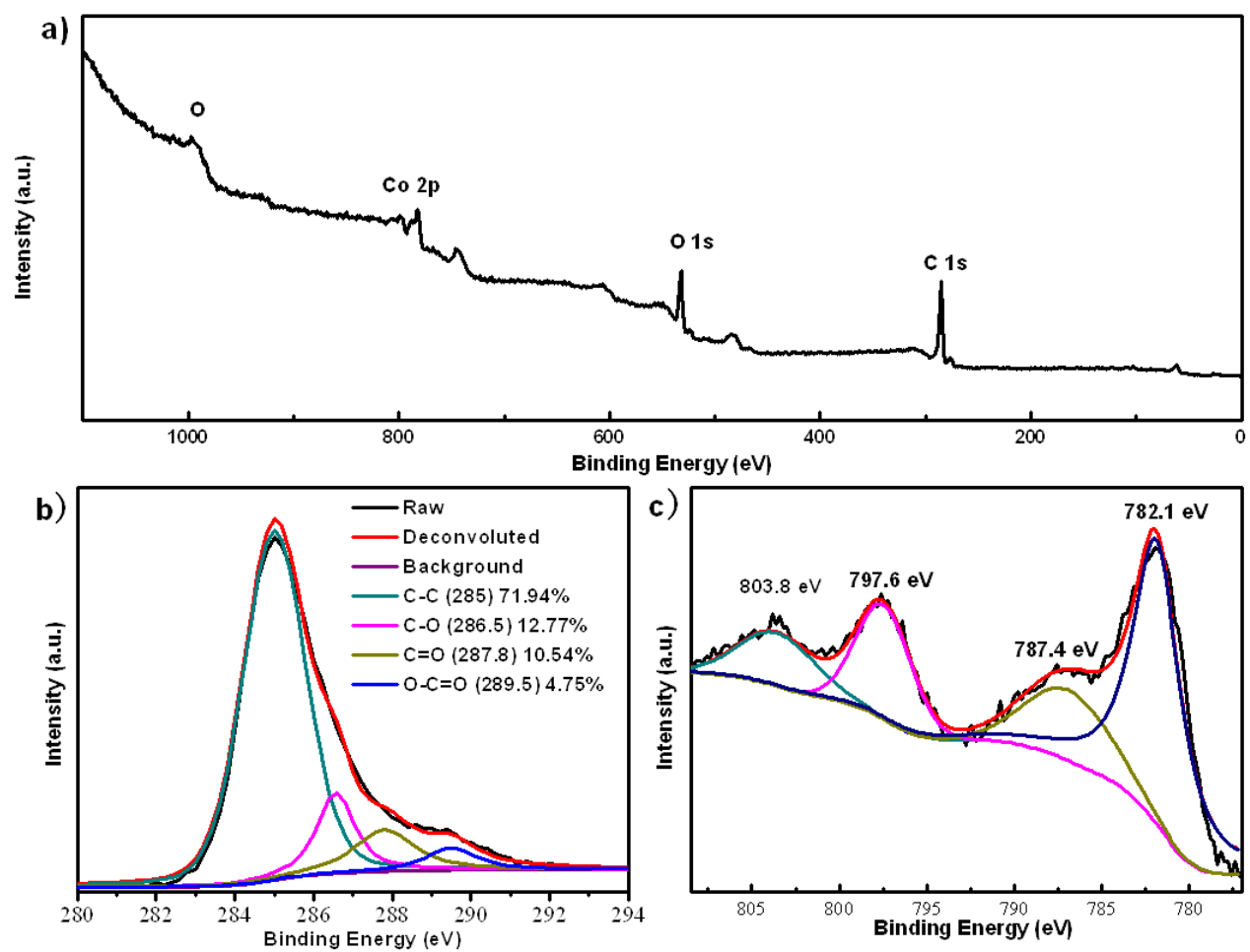

Fig 4. XPS spectra for the CoO/GS (15) composite: (a) survey spectrum and high-resolution (b) C $1 \mathrm{~s}$ and (c) Co $2 \mathrm{p}$ spectra. 


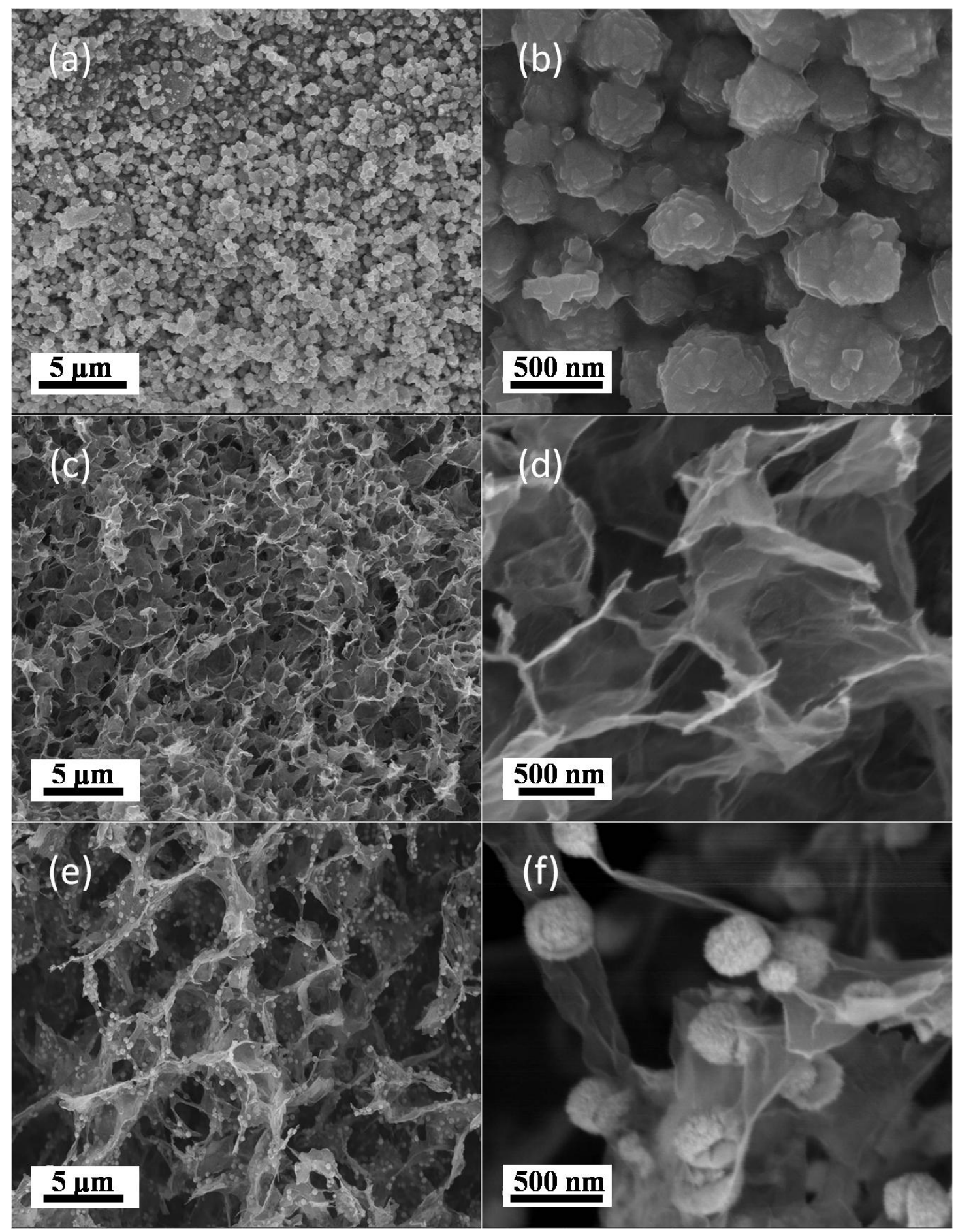

Fig. 5 FESEM images of (a,b) bare $\mathrm{CoO}$, (c,d) pristine GS, (e,f) CoO/GS (15). 


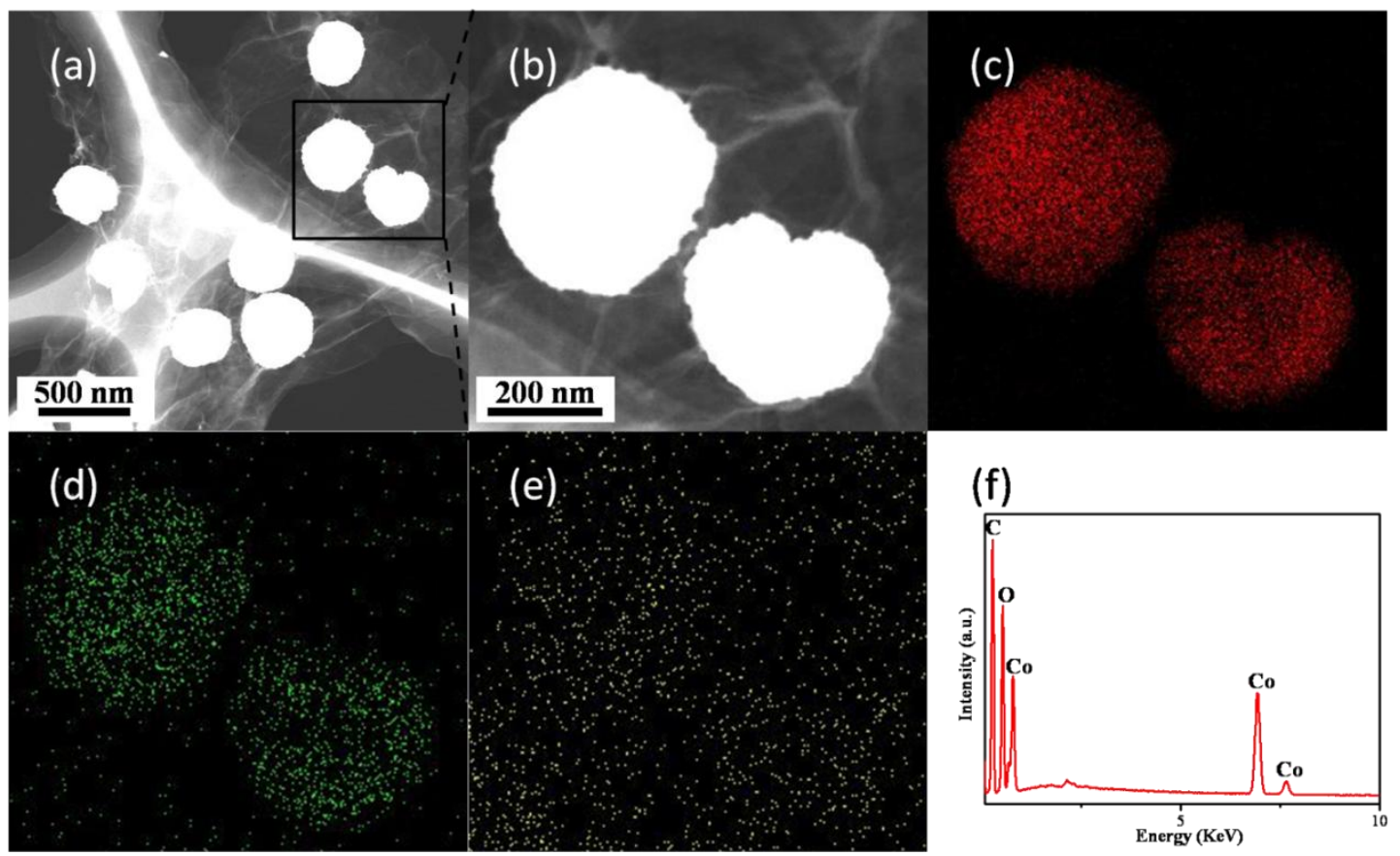

Fig. 6 STEM, Elemental mapping and EDX spectrum of CoO/GS (15). (a) Typical STEM image; (b) STEM image taken from the square region marked in (a) and corresponding elemental mapping images of (c) $\mathrm{Co}$, (d) $\mathrm{O}$ and (e) $\mathrm{C}$, and (f) EDS spectrum suggest the homogeneous distribution of $\mathrm{Co}, \mathrm{O}$ and $\mathrm{C}$ in $\mathrm{CoO} / \mathrm{GS}$ (15). 


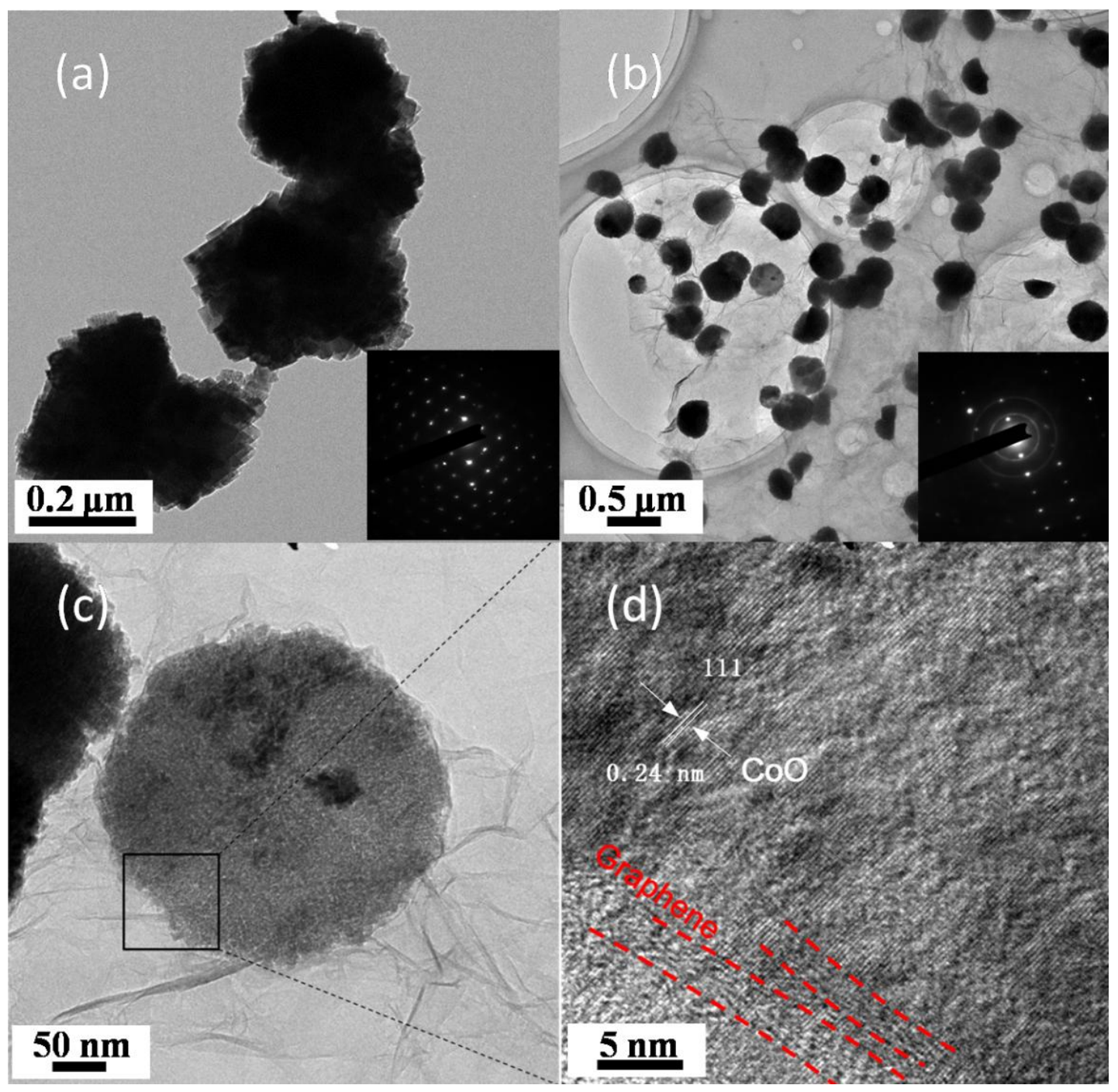

Fig. 7 TEM images of (a) bare CoO and (b-c) CoO/GS (15); HRTEM image of (d) CoO/GS (15). The insets in (a) and (b) are the electronic diffraction patterns of bare $\mathrm{CoO}$ and $\mathrm{CoO} / \mathrm{GS}$ (15), respectively 

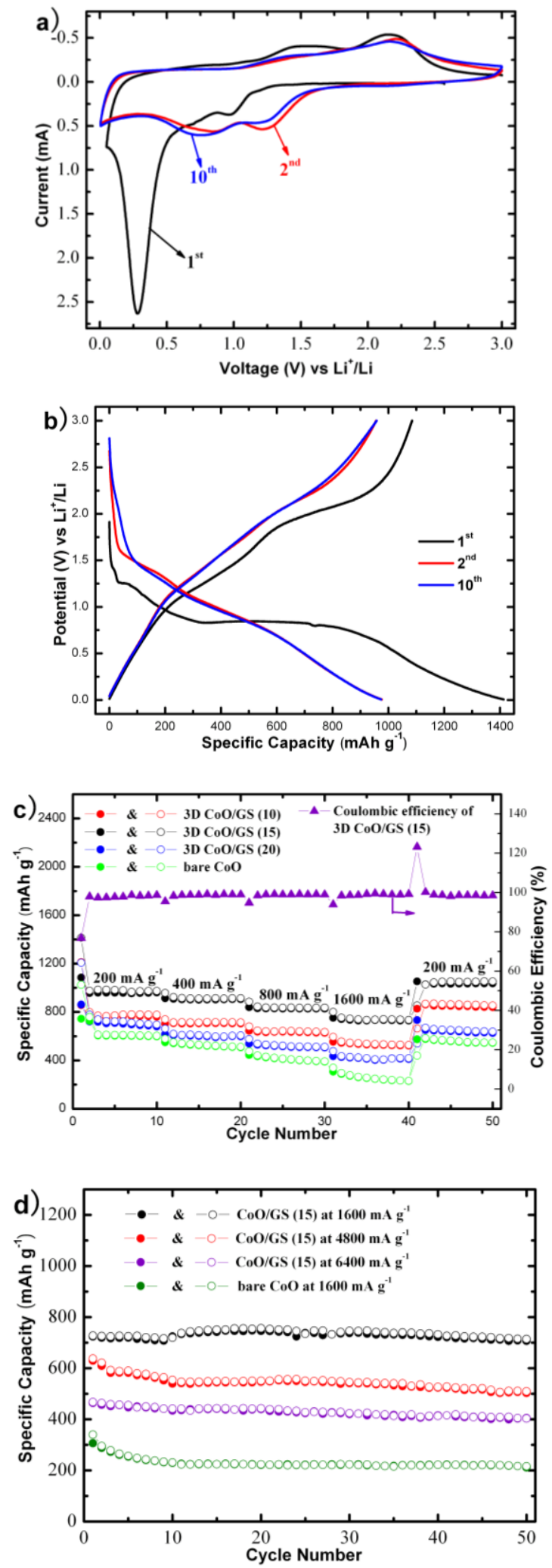

Fig. 8. (a) CV curves of the CoO/GS (15) composite in the potential range of $0.005-3 \mathrm{~V}$ at a scan rate of $0.5 \mathrm{mV} \mathrm{s}^{-1}$. (b) Charge-discharge curves of the CoO/GS (15) composite at a current density of $200 \mathrm{~mA} \mathrm{~g}^{-1}$. (c) Rate performance of the $\mathrm{CoO} / \mathrm{GS}$ composites with different graphene contents and bare $\mathrm{CoO}$ at various current densities. (d) Cycling behavior of bare $\mathrm{CoO}$ and $\mathrm{CoO} / \mathrm{GS}$ (15) at high current densities. Hollow and solid point symbols represent lithium insertion and extraction. 


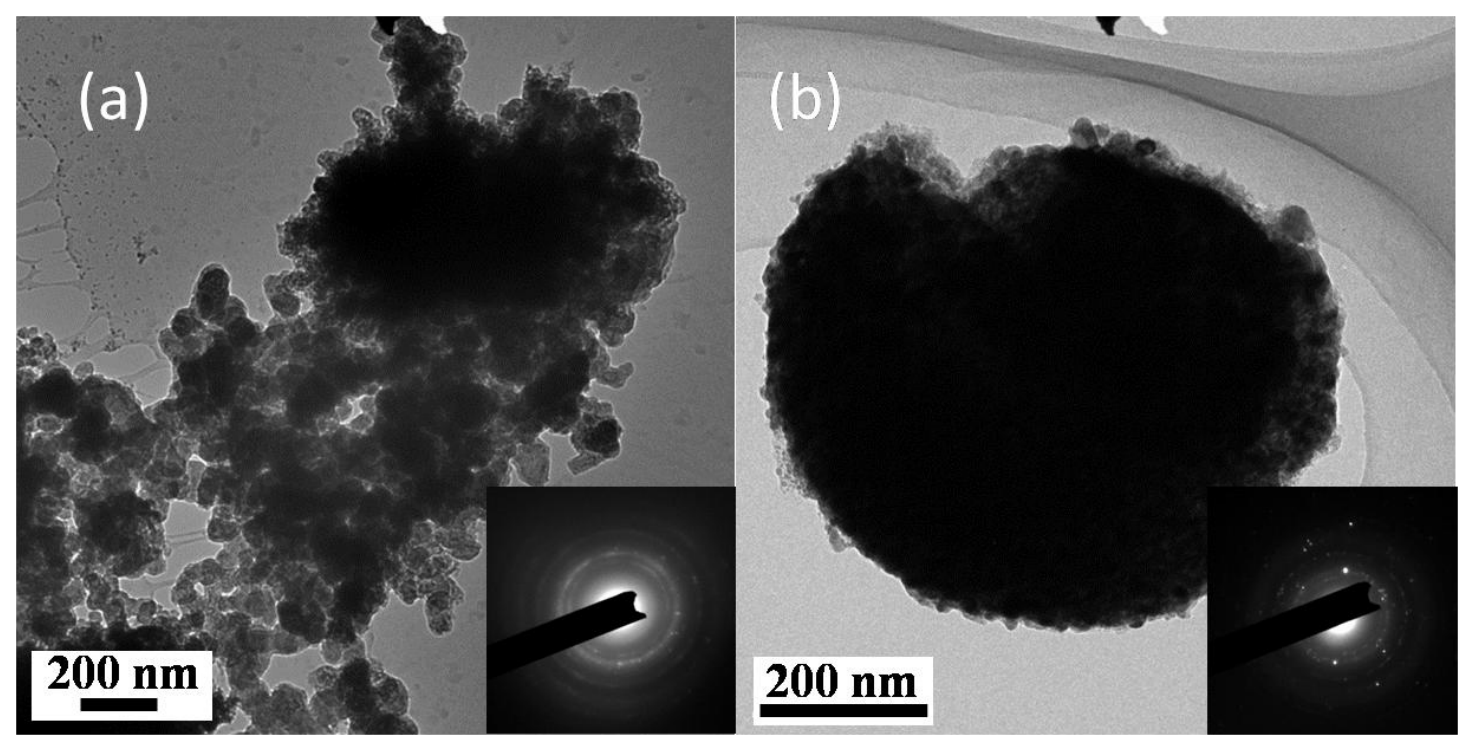

Fig. 9 TEM images of (a) bare $\mathrm{CoO}$ and (b) 3D CoO/GS (15) composite after 50 cycles at a current density of $1600 \mathrm{~mA} \mathrm{~g}^{-1}$. The insets in (a) and (b) are the corresponding electronic diffraction patterns. 